This item was submitted to Loughborough's Research Repository by the author.

Items in Figshare are protected by copyright, with all rights reserved, unless otherwise indicated.

\title{
The environment of safe care: Considering building design as one facet of safety
}

PLEASE CITE THE PUBLISHED VERSION

http://dx.doi.org/10.1177/2327857914031020

\section{PUBLISHER}

(c) Human Factors and Ergonomics Society. Published by Sage Journals

\section{VERSION}

AM (Accepted Manuscript)

\section{PUBLISHER STATEMENT}

This work is made available according to the conditions of the Creative Commons Attribution-NonCommercialNoDerivatives 4.0 International (CC BY-NC-ND 4.0) licence. Full details of this licence are available at: https://creativecommons.org/licenses/by-nc-nd/4.0/

\section{LICENCE}

CC BY-NC-ND 4.0

\section{REPOSITORY RECORD}

Taylor, Ellen, Sue Hignett, and Anjali Joseph. 2019. "The Environment of Safe Care: Considering Building Design as One Facet of Safety”. figshare. https://hdl.handle.net/2134/25392. 


\title{
The environment of safe care: considering building design as one facet of safety
}

\author{
Ellen Taylor, AIA \\ The Center for Health Design, Concord, CA, USA \\ Loughborough University, Loughborough, Leicestershire, UK \\ Sue Hignett, Ph.D. \\ Loughborough University, Loughborough, Leicestershire, UK \\ Anjali Joseph, Ph.D. \\ The Center for Health Design, Concord, CA, USA
}

\begin{abstract}
Patient safety is often considered in a behavioral context - what can someone do differently to improve outcomes? However, as a complex system of interactions, patient safety is better advanced through a systems thinking lens of human factors and ergonomics (HFE). While HFE is sometimes considered in three domains: physical, cognitive, and organizational, research in the area of the design of the physical environment is often limited to products, equipment and furnishings to accommodate a diverse population of users. With an increased focus on reimbursement related to patient safety as part of healthcare reform, organizations are becoming more aware of their own shortcomings and grappling with solutions to improve performance - typically people and processes. Yet the influence of the built environment, the space in which people work and are cared for, can act as a barrier or enhancement to achieving the desired results physically, cognitively, and organizationally. Latent conditions of the built environment can contribute to hazards and risk within the system and using Reason's Swiss Cheese Model can also become an additional layer of defense. A consensus-based safety risk assessment (SRA) design decision tool is being developed to address these built environment latent conditions funded through a three-year grant from the Agency for Healthcare Research and Quality (AHRQ).
\end{abstract}

\section{BACKGROUND}

Patient safety is often considered in a behavioral context what can someone do differently to improve outcomes? However, as a complex system of interactions, patient safety is better advanced through a systems thinking lens of human factors and ergonomics (HFE). While HFE is sometimes considered in three domains: physical, cognitive, and organizational, research in the area of the design physical environment is often limited to products, equipment and furnishings that are designed to accommodate a diverse population of users.

With an increased focus on reimbursement related to patient safety as part of healthcare reform, organizations are becoming more aware of their own shortcomings and grappling with solutions to improve performance - typically people and processes. Yet the influence of the built environment, the space in which people work, can act as a barrier or enhancement to achieving the desired results physically, cognitively, and organizationally. Latent conditions of the built environment can contribute to hazards and risk within the system and, using Reason's Swiss Cheese Model (1990), the built environment can also become an additional layer of defense.

Accidents happen when holes in the system align. While active failures may be difficult to anticipate, latent conditions can be proactively identified as preconditions for error before an accident occurs (Lawton et al., 2012; Reason, 1990). This framework has been further developed (Figure 1) to include the role of the built environment as a latent condition with considerations for design factors such as for air quality; room occupancy; patient room design; lighting, interior design, noise, unit layout, and access to hand hygiene (Henriksen, Joseph, \& Zayas-Caban, 2009; Joseph \& Malone, 2012; Joseph \& Rashid, 2007; Reiling, Hughes, \& Murphy, 2008).

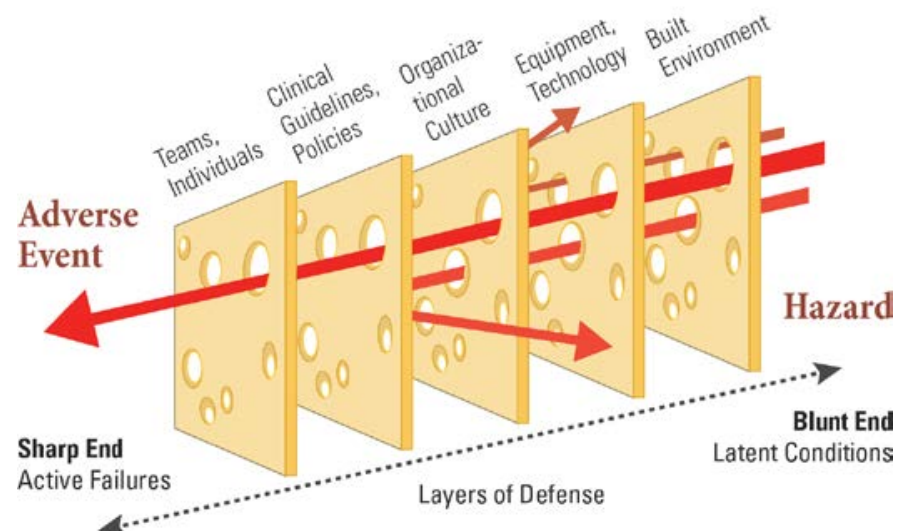

Figure 1: Reason's Swiss Cheese Model(Adapted from Reason, 1990)

In a paper suggesting HFE principles in design, Attaianese and Duca (2012) state, "when the system is the built environment, the systemic approach requires that designers move from an attention exclusively reserved for building functions towards the set of actions that users actually perform and that building has to support." 


\section{The Cost-Influence Curve}

Because each stage in the design process includes decisions that carry forward through the project, changes to previously approved decisions incur additional cost. The further into the project schedule, the more expensive changes become (Figure 2). Since the budget is often established before the project is designed, most of these decisions are made in the earliest phases of project planning, before a design team has been engaged. If safety is not considered a priority during these early phases, it is likely that features to support the safe delivery of care will not be included.

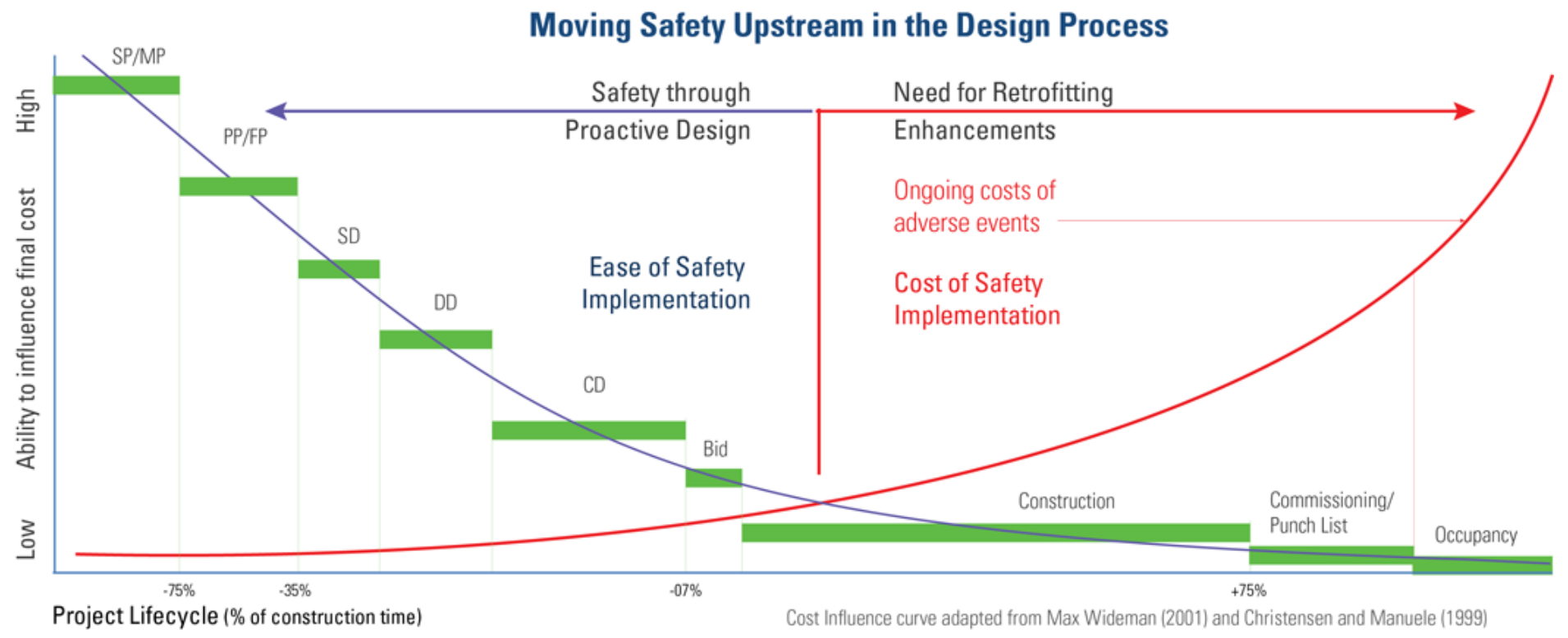

Figure 2: The Cost-Influence Curve (Adapted from Wideman, 2001 and Christensen and Manuele, 1999)

\section{A Safety Risk Assessment for the Built Environment}

Healthcare architecture has become an increasingly specialized field, marked by a complex interaction between people, operations and the physical environment and an ever changing landscape of regulation and reimbursement. Recognizing this, Safety Risk Assessment (SRA) language will be included in the 2014 FGI Guidelines for the Design and Construction of Hospitals and Outpatient Facilities. Multiple risks are to be considered as underlying conditions to injury or harm in the design of healthcare environments. These include: infection control and hospital associated infections (HAI), falls and immobility, medication errors and safety, patient handling, security, and behavioral health/psychiatric injury.

\section{METHODS}

Processes such as evidence-based design strive to engage teams in considering the best available research to optimize outcomes, but the use of standardized tools to facilitate a proactive thought process is rare. Funded through a three-year grant from the Agency for Healthcare Research and Quality, a SRA design decision tool is being developed to address these built environment latent conditions. This leverages work completed in 2010 surrounding the topic of design for patient safety, in which a number of methods were identified as potential processes that could be used during the building design process (Taylor, Joseph, \& Quan, 2012). The criteria for selection of methods included the relevance to healthcare facility design, scope of use within healthcare, documented effectiveness, and tool validity and excluded high-level design approaches or philosophies (e.g., lean). The evaluated methods included: link analysis, root cause analysis, failure modes and effects analysis (FMEA), simulation, work sampling, balanced scorecard, and process analysis.

\section{Literature Reviews}

As part of a $\mathrm{PhD}$, a literature review was conducted to better understand the considerations for development of the SRA tool. The goal was to not only identify development of specific tools, but to understand how (or if) the tools successfully interface with stakeholders and the types of issues that might warrant consideration in tool development. Reviewed tools included: Achieving Excellence Design Evaluation Toolkit (AEDET) Evolution (being supplemented by the exemplary layer) (University of Sheffield, 2007); the Government of Alberta Building Performance Evaluation (BPE) (Steinke, Webster, \& Fontaine, 2010); a Sharing Knowledge "manual” (Bartholomew, 2005); the Design Quality Indicator (DQI) (Gann, Salter, \& Whyte, 2003; Whyte \& Gann, 2003); Environmental Audit Tool (EAT) (Smith et al., 2012); the US Military Health System (MHS) PostOccupancy Evaluation (POE) and World-Class Checklist 
(Clemson \& NXT, 2012); Usable Buildings Portfolio (Bordass \& Leaman, 2005); and the Physical Security Review Checklist (MacAlister, 2013).

The literature review resulted in a model based on prior work by Hignett and Lu (2009) consisting of several influences: design culture, the evidence based, and guidance needs (Figure 3). New subthemes were developed within these three themes. Design culture included two sub-factors: existing processes (questions surrounding design value, healthcare design context, education paradigms, established steps and tasks in the traditional design process, and the design climate that often includes competing priorities and drivers) and systems thinking (the behavior-environment interaction, user input and participatory design, and organizational and design team silos). The evidence base included three subthemes: using, sharing, and managing knowledge. Using knowledge takes into account learning styles, the range of information and the ability to synthesize information, while sharing information considers information availability, reactive processes and the opportunity for proactive assessments. Managing knowledge reviews the systems for collection and classification, translation, and the ability to maintain and sustain knowledge management systems. Lastly, guidance need subthemes included: direction (benchmarking and integration of the human response); change (education awareness, filters and gates to validate information, communication and feedback loops, and interface with codes and regulations); and tools (development, testing and piloting of design tools).

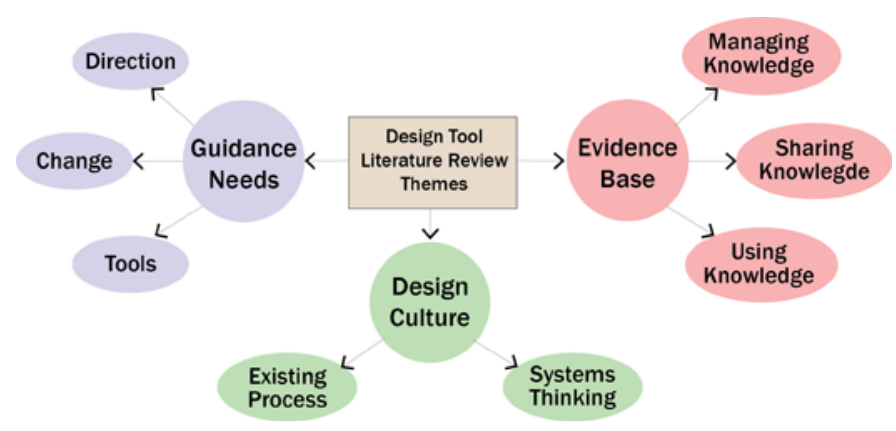

Figure 3: Thematic Model for Design Tool Development

The review reveals the importance of considering safety as a goal early in the design process; challenges associated with decisions confounded by competing drivers; compromises when conflicting strategies are introduced; and the fallacy that most likely exists in the traditional user-input process for architectural design.

Topic specific literature reviews for the SRA considerations were conducted by the research team at The Center for Health Design. This included searches for: research (empirical research and literature reviews), consensus documents (white papers and recognized consensus-based design guidelines), or other (expert opinion). These were gathered through searches of several sources, such as The Center for Health Design Knowledge Repository (http://www.healthdesign.org/search/articles), PubMed, and Google Scholar. Sources were indexed for such items as study design, setting, and outcomes. Outcomes were categorized based upon the development of an evidence-based design (EBD) glossary produced in 2011 by The Center for Health Design. A single built-environment study feature (e.g. flooring) was often difficult to identify due the multifactorial solutions referenced in the papers. A second systematic review was conducted for falls, appraising the available evidence (Taylor \& Hignett, 2014).

\section{Tool Development through Consensus}

The tool development was envisioned as a multidisciplinary collaborative process using subject matter experts with diverse backgrounds to evaluate the SRA content. Workgroup leaders for the six risk components were established and volunteers (10-20 per group) were recruited for workgroups to represent a diversity of expertise and views for each particular topic area (Figure 4).

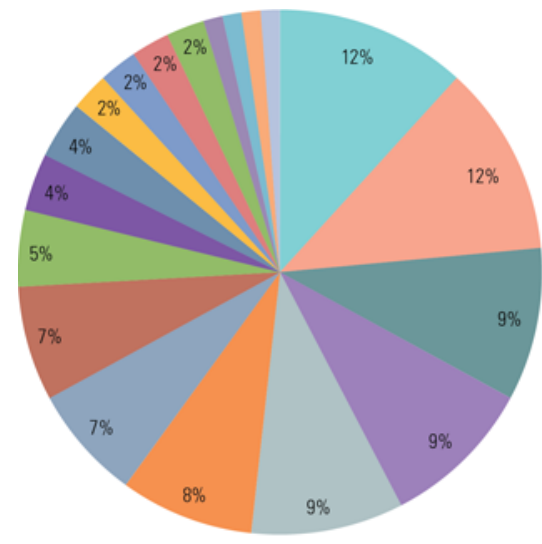

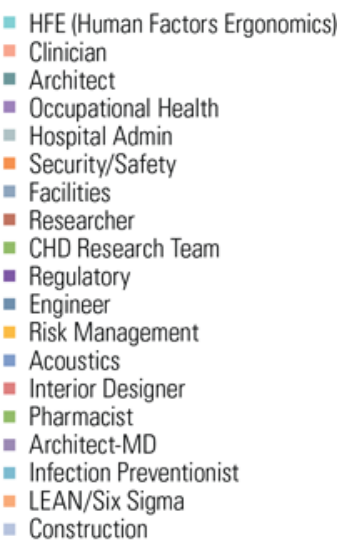

Figure 4: SRA Content Development Participants

A modified Delphi process was used to gain consensus from workgroup participants for content relevant to each risk area. A first questionnaire for the Delphi process was developed based upon the literature review for each of the hazard topics and distributed online (Survey Monkey). A second online questionnaire was distributed based upon the results of the first questionnaire. Items that obtained consensus (70 percent) were not included in the subsequent Delphi rounds, and new wording was proposed for items reaching consensus for inclusion, but not in wording. Following the second online survey, the items that had not achieved consensus were brought forward to the face-to-face seminar held in June 2013. A modified nominal group technique was used at the seminar to achieve final consensus. The consensus flow is shown in Figure 5.

\section{RESULTS}

The process to date has resulted in 200 consideration statements (and contextual rationale) across the risk components to prompt thought and discussion among diverse design team stakeholders (Table 1). These considerations are supplemented by a Safe Design Roadmap, with questions that identify opportunities to further engage the team in 
considering the interaction between the healthcare facility environment, safety outcomes, performance improvement, and a leadership-led safety culture. The tool is currently in the pilot testing phase (Year 2 of 3). This will include evaluation at three project sites with design teams and hospital stakeholders, as well as case-study evaluations planned at a second face-to-face seminar.

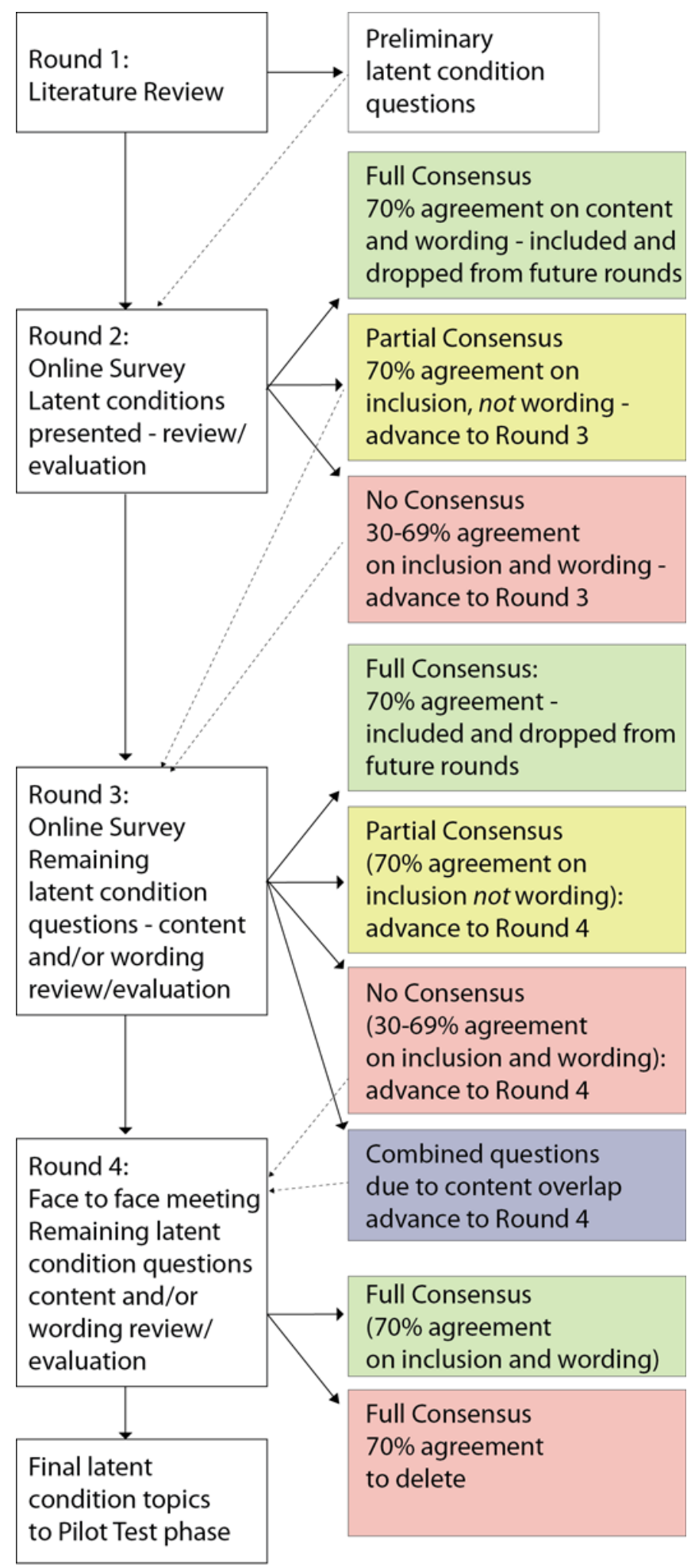

Figure5: Content Development Consensus Flow

\begin{tabular}{|l|l|}
\hline Topic Area & $\begin{array}{l}\text { Consensus Considerations } \\
\text { following Year 1 }\end{array}$ \\
\hline HAI & $32 ; 12$ additional to be revisited \\
\hline Patient handling & 21 \\
\hline Medication safety & 30 \\
\hline Security & 47 \\
\hline Falls and immobility & 32 \\
\hline $\begin{array}{l}\text { Behavioral health and } \\
\text { psychiatric injury }\end{array}$ & 54 \\
\hline
\end{tabular}

Table 1: Topic Area and Current Content Considerations

\section{CONCLUSION}

The focus of the SRA tool is to promote discussion for built-environment decision-making, but healthcare facility design is inextricable linked to people (patients and staff), operations and organizational policy. The interaction of other components that create "the system" should be recognized as components that need additional evaluation. Engagement of stakeholders who are able to contribute understanding through a human factors and ergonomics perspective would benefit the process. The tool cannot change the design process singlehandedly, but it may take a step forward in a more holistic understanding of the actions and processes of users that the building is intended to support.

Portions of this project were supported by grant number R13HS021824 from the Agency for Healthcare Research and Quality. The content is solely the responsibility of the author and does not necessarily represent the official views of the Agency for Healthcare Research and Quality.

\section{REFERENCES}

Attaianese, E., \& Duca, G. (2012). Human factors and ergonomic principles in building design for life and work activities: an applied methodology. Theoretical Issues in Ergonomics Science, 13(2), 187-202. doi: 10.1080/1463922X.2010.504286

Bartholomew, D. (2005). Sharing Knowledge. Cheltenham, Gloucestershire: DBA (David Bartholomew Associates).

Bordass, B., \& Leaman, A. (2005). Making feedback and postoccupancy evaluation routine 1: A portfolio of feedback techniques. Building Research \& Information, 33(4), 347-352. doi: $10.1080 / 09613210500162016$

Clemson, U., \& NXT. (2012). Pathway towards the Development of a Post Occupancy Evaluation (POE) Program and Policy for the Military Health System (pp. 115): Military Health System/Department of Defense.

Gann, D., Salter, A., \& Whyte, J. (2003). Design Quality Indicator as a tool for thinking. Building Research \& Information, 31(5), 318-333. doi: 10.1080/0961321032000107564

Henriksen, K., Joseph, A., \& Zayas-Caban, T. (2009). The human factors of home health care: a conceptual 
model for examining safety and quality concerns. $J$

Patient Saf, 5(4), 229-236. doi:

10.1097/PTS.0b013e3181bd1c2a

Hignett, S., \& Lu, J. (2009). An investigation of the use of health building notes by UK healthcare building designers. Applied Ergonomics, 40(4), 608-616. doi: 10.1016/j.apergo.2008.04.018

Joseph, A., \& Malone, E. (2012, 2012/10//

2013/09/29/18:05:23). The Environment: An Often Unconsidered Patient Safety Tool. AHRQ WebM\&M: Morbidity and Mortality Rounds on the Web. from http://webmm.ahrq.gov/perspective.aspx?perspective $\underline{\mathrm{ID}=130}$

Joseph, A., \& Rashid, M. (2007). The architecture of safety: hospital design. Curr Opin Crit Care, 13(6), 714-719. doi: 10.1097/MCC.0b013e3282f1be6e

Lawton, R., McEachan, R. R., Giles, S. J., Sirriyeh, R., Watt, I. S., \& Wright, J. (2012). Development of an evidence-based framework of factors contributing to patient safety incidents in hospital settings: a systematic review. BMJ Qual Saf, 21(5), 369-380. doi: 10.1136/bmjqs-2011-000443

MacAlister, D. (2013). A physical security evaluation tool for elopement prevention in a behavioural/mental health setting. (0891-7930 (Print)).

Reason, J. (1990). The contribution of latent human failures to the breakdown of complex systems. Philos Trans $R$ Soc Lond B Biol Sci, 327(1241), 475-484.

Reiling, J., Hughes, R. G., \& Murphy, M. R. (2008). The Impact of Facility Design on Patient Safety. Patient Safety and Quality: An Evidence-Based Handbook for Nursing. Rockville, MD: Agency for Healthcare Research \& Quality.

Smith, R., Fleming, R., Chenoweth, L., Jeon, Y.-H., SteinParbury, J., \& Brodaty, H. (2012). Validation of the Environmental Audit Tool in both purpose-built and non-purpose-built dementia care settings.

Australasian Journal on Ageing, 31(3), 159-163. doi: 10.1111/j.1741-6612.2011.00559.x

Steinke, C., Webster, L., \& Fontaine, M. (2010). Evaluating building performance in healthcare facilities: an organizational perspective. HERD, 3(2), 63-83.

Taylor, E., \& Hignett, S. (2014). Evaluating Evidence: Defining Levels and Quality through Existing Methods. HERD, 7(3), in press.

Taylor, E., Joseph, A., \& Quan, X. (2012). Designing for Patient Safety - Considering a Patient Safety Risk Assessment Advances in Human Aspects of Healthcare (Vol. 3, pp. 249-258). Purdue University, Indiana: CRC Press.

University of Sheffield, S. o. A. (2007). Disseminating good practice (DGP): developing an exemplar layer for AEDET Evolution and ASPECT design evaluation tools. United Kingdom: NHS Estates, Department of Health.

Whyte, J., \& Gann, D. (2003). Design Quality Indicators: work in progress. Building Research \& Information, 31(5), 387-398. doi: 10.1080/0961321032000107537 